\title{
Effectiveness and Safety of Cytoreduction Surgery in Advanced Ovarian Cancer: Initial Experience at a University General Hospital
}

\author{
Jose Antonio Llueca ${ }^{a}$, d, Jose Luis Herraiz ${ }^{\mathrm{a}}$, Carmen Catala ${ }^{\mathrm{a}}$, Anna Serra ${ }^{\mathrm{a}}$, \\ Isabel Rivadulla $^{\mathrm{b}}$, Javier Escrig ${ }^{\mathrm{b}}$, MUAPOS Working Group ${ }^{\mathrm{c}}$
}

\begin{abstract}
Background: The aim of the study was to evaluate the initial efficiency and security of maximal effort cytoreductive surgery in stages III and IV of ovarian cancer at a university hospital.

Methods: Thirty-four patients with stage III and IV ovarian carcinoma underwent surgery between January 2013 and June 2014 in the University General Hospital of Castellon (Spain). Patients with primary and relapse ovarian cancer were included. The extent of disease, type of surgical technique, amount of tumor prior to surgery and amount of residual disease after surgery were recorded. To quantify the efficiency and the security of the procedure, the complete cytoreduction and the morbidity and the mortality rates were described.
\end{abstract}

Results: Of the patients $26.4 \%$ received neoadjuvant chemotherapy. Complete cytoreductive surgery, without evident residual tumor at the end of the procedure, was obtained in $79 \%$ of patients and optimal cytoreductive surgery $(\mathrm{CC} 0-\mathrm{CC} 1)$ in $91 \%$. Surgical complications were found in $56 \%$ of patients and two deaths $(6 \%)$ occurred attributable to surgery. The disease free interval was 15 months.

Conclusions: This study confirms that with experienced multidisciplinary teams and in tertiary referral hospitals, 79\% of complete cytoreduction surgery in advanced ovarian cancer can be achieved but they must be prepared to deal with a high rate of complications.

Keywords: Cytoreductive surgery; Advanced ovarian cancer; Morbidity; Mortality; Ovary; Cancer; Surgery

Manuscript accepted for publication July 09, 2015

a Deparment of Obstetrics and Gynecology, University General Hospital of Castellon, Dept. of Medicine, University Jaume I, Castellon de la Plana, Spain bDepartment of General Surgery, University General Hospital of Castellon, Dept. of Medicine, University Jaume I, Castellon de la Plana, Spain

${ }^{c}$ Multidisciplinary Unit of Abdominal Pelvic Oncology Surgery

${ }^{\mathrm{d} C}$ Corresponding Author: Jose Antonio Llueca Abella, Av Benicasim, 12004 ,

Castellon, Spain. Email: antonillueca@gmail.com

doi: http://dx.doi.org/10.14740/jcgo345w

\section{Introduction}

For patients with advanced epithelial ovarian cancer, the amount of post surgery residual tumor constitutes the most important prognostic factor related to survival. The survival rate of 5 years reaches almost $50 \%$ for patients with a null residual tumor after surgery and diminishes to $5 \%$ in patients with residual tumor $>1-2 \mathrm{~cm} \mathrm{[1].}$

Therefore we can ensure that surgery is considered essential for treatment of advanced epithelial ovarian cancer. In general, this cytoreduction surgery (CS) is used initially in advanced cases of ovarian cancer including stage IV when metastatic lesions present at a distance that does not influence survival in the short run. This surgery of cytoreduction ought to attempt to remove all metastatic and spreading lesions of the ovarian tumor through the abdominal cavity, such that the residual tumor should be reduced to zero upon visual inspection. Only when we leave a residual tumor of $<1 \mathrm{~cm}$, optimal surgery of cytoreduction (OSC), does the prognosis of the patient improve [2].

As previously mentioned, survival of advanced ovarian cancer in Castellon (Spain) is low [3]. Recently multidisciplinary techniques of abdominal surgery have been adopted as part of the CS. This study analyzes the initial results as much in terms of safety as well as in effectiveness in the treatment of advanced ovarian cancer at a tertiary referral hospital for patients with gynecological cancer.

\section{Material and Methods}

From January 2013 to June 2014, a total of 34 patients with advanced ovarian epithelial cancer have taken part in the Multidisciplinary Unit of Abdominal Pelvic Oncology Surgery (MUAPOS) at the University General Hospital of Castellon (Spain); of these patients 22 presented with primary tumors and 12 as relapsed. All procedures were carried out by the same surgical team.

The World Health Organization Classification was used for the histological classification [4]. The histological degree was assessed according to the criteria of Day et al [5]. Staging was determined by the FIGO system [6]. All patients were prepared with the normal colon Citrafleet ${ }^{\mathbb{R}}$ the day before the procedure. Standard antibiotic prophylactics of metronidazol 1,500 $\mathrm{mg} \mathrm{EV}$ and gentamicin $240 \mathrm{mg} \mathrm{EV}$ were used the day

Articles (C) The authors | Journal compilation (C) J Clin Gynecol Obstet and Elmer Press Inc ${ }^{\mathrm{TM}}$ | www.jcgo.elmerpress.com

This is an open-access article distributed under the terms of the Creative Commons Attribution License, which permits unrestricted use, distribution, and reproduction 


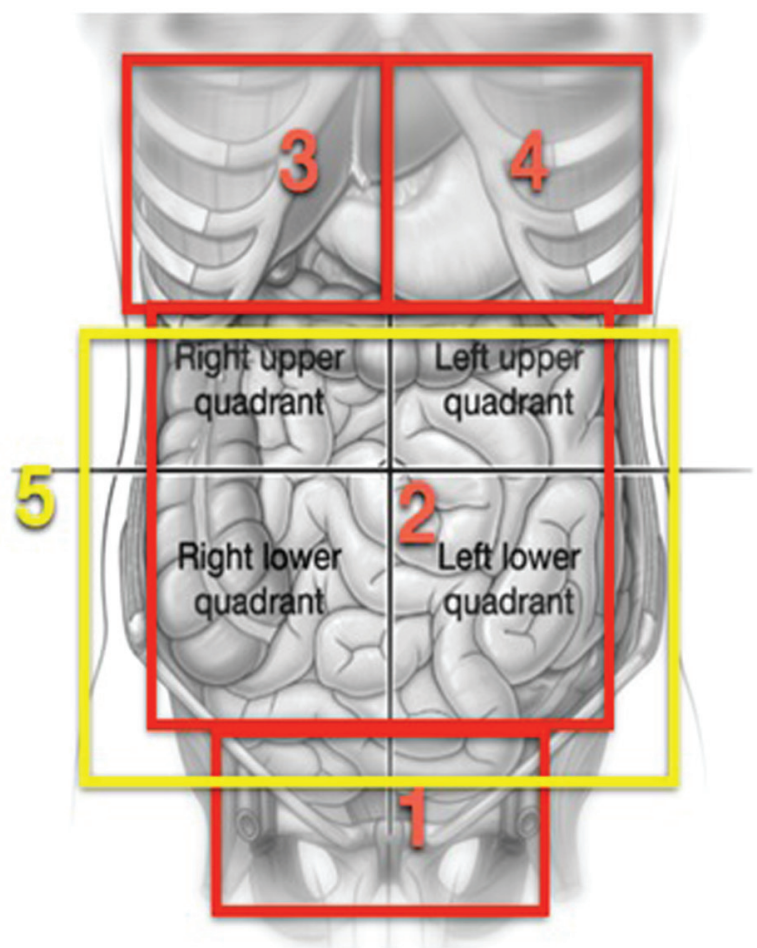

Figure 1. Graphs reproducing areas described in the process proposed by Morrow [8].

of the procedure as well as a dose of amoxycillin-clavulanic $2 \mathrm{~g} \mathrm{EV}$ at $4 \mathrm{~h}$ into surgery. In addition, a thromboprophylaxis with heparin of low molecular weight at a standard dose was administered and was continued during the post operation period, and pneumatic compression stockings were put in place until the patient was able to walk.

All these procedures were carried out under general anesthesia and by means of a laparotomy xifo-pubic incision. The index of peritoneal carcinomatosis (IPC) was calculated according to Sugarbaker [7]. Surgery by cytoreduction for advanced ovarian cancer is composed of multiple procedures during a single operation, thus it was divided into several steps during which different abdominal pelvic regions were worked on, following the outline proposed by Morrow [8] (Fig. 1).

In the first area, the abdominal pelvic, routine procedures in gynecological oncology such as hysterectomies and adnexectomies are carried out, but in a large number of the patients, due to a large quantity of tumors found in the pelvis, a resection is done in bloc of the uterus, ovaries, pelvic peritoneum, resection of associated tumor nodules, and of the rectum as part of the pelvic CS (radical pelvic peritonectomy). The procedure is carried out following the process described by Morrow [8]. Briefly, after opening the retroperitoneal spaces, infundibulopelvic ligaments are cut open and the arteries and ovarian veins are tied and a bilateral ureterolysis is carried out. The incisions are lengthened through the pararectal passage and are penetrated in the retrorectal spaces. The upper rectal vessels are cut open as low as possible but above the site of the cancer, a bilateral ureterolysis is carried out. The incisions are stretched
Table 1. Characteristics of the Patients and of the Illness

\begin{tabular}{|c|c|}
\hline & $\mathrm{n}=\mathbf{3 4}$ \\
\hline Age & $60(41-84)$ \\
\hline \multicolumn{2}{|l|}{ Tumor } \\
\hline Primary & $22(65 \%)$ \\
\hline Recurrence & $12(35 \%)$ \\
\hline Serous/Papillary-Serous & $14(41 \%)$ \\
\hline Endometrioid & $5(15 \%)$ \\
\hline Granular & $1(3 \%)$ \\
\hline Mullerian & $1(3 \%)$ \\
\hline Neuroendocrine & $1(3 \%)$ \\
\hline Mixed & $1(3 \%)$ \\
\hline Peritoneal & $9(26 \%)$ \\
\hline Indistinct & $2(6 \%)$ \\
\hline \multicolumn{2}{|l|}{ FIGO } \\
\hline IIIC & $18(53 \%)$ \\
\hline IV & $16(47 \%)$ \\
\hline \multicolumn{2}{|l|}{ ASA } \\
\hline I & $1(3 \%)$ \\
\hline II & $9(26 \%)$ \\
\hline III & $23(68 \%)$ \\
\hline IV & $1(3 \%)$ \\
\hline IPC operative & $12(2-33)$ \\
\hline $1-10$ & $13(38 \%)$ \\
\hline $11-20$ & $13(38 \%)$ \\
\hline 20 & $8(24 \%)$ \\
\hline Primary & $14(4-33)$ \\
\hline Recurrence & $5(2-24)$ \\
\hline CEA & $2(1-150)$ \\
\hline CA199 & $17(3-67)$ \\
\hline CA125 & $254(24-14,125)$ \\
\hline CA153 & $115(5-1,500)$ \\
\hline Ascites (clinically evident) & $9(26 \%)$ \\
\hline Pleural spillage (radiological) & $7(21 \%)$ \\
\hline Intestinal subocclusion & $4(12 \%)$ \\
\hline Primary & $2(9 \%)$ \\
\hline Recurrence & $2(17 \%)$ \\
\hline Preoperative laparoscopy & $19(56 \%)$ \\
\hline IPC $1-10$ & $6(18 \%)$ \\
\hline IPC $11-20$ & $11(32 \%)$ \\
\hline $\mathrm{IPC}+20$ & $2(6 \%)$ \\
\hline IPC $1-10$ accuracy & $33 \%$ \\
\hline IPC +10 accuracy & $63 \%$ \\
\hline
\end{tabular}

Quantitative variables: median (range). Nominal variables: count (\%). 
Table 2. Treatment and Results

\begin{tabular}{|c|c|}
\hline & $n=34$ \\
\hline Visceral resections & $30(88 \%)$ \\
\hline \multicolumn{2}{|l|}{ Visceral resections per patient } \\
\hline 0 & $5(15 \%)$ \\
\hline 1 & $1(3 \%)$ \\
\hline 2 & $9(26 \%)$ \\
\hline 3 & $5(15 \%)$ \\
\hline 4 & $3(9 \%)$ \\
\hline 5 & $2(6 \%)$ \\
\hline 7 & $5(15 \%)$ visceral resections \\
\hline 8 & $2(6 \%)$ \\
\hline 11 & $1(3 \%)$ \\
\hline 14 & $1(3 \%)$ \\
\hline \multicolumn{2}{|l|}{ Peritonectomy (all) } \\
\hline Complete & $21(62 \%)$ \\
\hline Partial & $7(21 \%)$ \\
\hline No & $6(17 \%)$ \\
\hline \multicolumn{2}{|l|}{ Primary } \\
\hline Complete & $18(82 \%)$ \\
\hline Partial & $3(14 \%)$ \\
\hline No & $1(4 \%)$ \\
\hline \multicolumn{2}{|l|}{ Relapse } \\
\hline Complete & $3(25 \%)$ \\
\hline Partial & $4(33 \%)$ \\
\hline No & $5(42 \%)$ \\
\hline Duration of surgery (min) & $540(280-750)$ \\
\hline Primary & $540(390-750)$ \\
\hline Relapse & $480(280-720)$ \\
\hline Regulated lymphadenectomies & $27(80 \%)$ \\
\hline Lymph nodes analyzed & $26(1-88)$ \\
\hline Lymph nodes positive & $2(0-36)$ \\
\hline $\begin{array}{l}\text { Infrarenal aorta caval } \\
\text { lymphadenectomy }\end{array}$ & $27(80 \%)$ \\
\hline Lymph nodes analyzed & $12(1-28)$ \\
\hline Lymph nodes positive & $1(0-12)$ \\
\hline Left iliac pelvic lymphadenectomy & $23(66 \%)$ \\
\hline Lymph nodes analyzed & $7(1-29)$ \\
\hline Lymph nodes positive & $1(0-11)$ \\
\hline $\begin{array}{l}\text { Right iliac pelvic } \\
\text { lymphadenectomy }\end{array}$ & $22(65 \%)$ \\
\hline Lymph nodes analyzed & $7(1-19)$ \\
\hline Lymph nodes positive & $1(0-15)$ \\
\hline Supsramesocolic lymphadenectomy & $5(15 \%)$ \\
\hline Lymph nodes analyzed & $2(1-9)$ \\
\hline Lymph nodes positive & $1(0-2)$ \\
\hline
\end{tabular}

along the pararectal passage and enter into the recto rectal spaces. The superior rectal vessels are cut open as low as possible but above the site of the cancer, the sigmoid colon is cut open with stapler type GIA (Multifire GIA, Auto Suture, Nor-Walk, $\mathrm{CT}$ ). The rectum is mobilized from the sacrum directing the dissection towards the pelvic diaphragm and the middle rectal arteries are cut open and tied. The inferior mesenteric artery is not cut open as normally done, except when it is necessary to mobilize the descending colon to achieve a low collateral anastomosis without tension. A retrograde hysterectomy is carried out and the rectum is cut open with a stapler type TA (Multifire TA Disposable Stapler, Auto Suture), beneath the peritoneal reflection at some $10 \mathrm{~cm}$ of the anal margin. This procedure as described allows for the resection in-bloc of all pelvic matter. In cases where a complete-terminal anastomosis was carried out, this was done with an end-stapler EEA (Premium Plus CEEA 28 to $33 \mathrm{~mm}$, Auto Suture). At the halfway point of the operation, efforts are dedicated to the upper abdomen. Thus, in zone 3, which corresponds to the right hypochondrium, procedures such as diaphragmatic peritonectomy, resection of the diaphragmatic muscle, glissectomy, cholecystectomy or hepatectomy are performed as is required per the site of the cancer. In zone 4, diaphragmatic peritonectomy, diaphragm resections, splenectomy with or without distal pancreatectomy are done, if the cancerous site requires it. In zone 2 procedures on the stomach, gastrocolic ligaments, upper and lower omentum, duodenum, ileum and colon are also performed. Finally in zone 5 , attention is focused on the aortocaval and supramesocolic lymph nodes and in the resection of macroscopically swollen lymph nodes when found.

Complications are classified according to the degrees of Clavien-Dindo [9] and only those with moderate to high severity (II and III degrees) are taken into consideration. The patients are released from the hospital when they are able to tolerate food; they recover bladder and intestinal functions, and they are afebrile.

As primary statistical parameters for analysis, the effectiveness parameters are considered (resectability: complete peritonectomies and total cytoreduction) and safety (morbidity and mortality).

Quantitative variables are described by the mean \pm standard deviation, or with the median (range) according to the outcomes. Qualitative variables are characterized by count and percentage. The proof of Mann-Whitney, the exact proof as Fisher, is used for the univariate analysis, and the simple lineal regression, according to the type of variables. For calculations of rates of survival, Kaplan-Meier's method is used. In order to calculate free of cancer survival rates a recurrence is considered when the tumor reappears again in the imaging tests along with tumoral markers.

\section{Results}

Analysis is based on the results obtained from 34 patients. The main characteristics of these patients are shown in Table 1. Neoadjuvant was administered in primary tumors in nine patients $(26 \%)$. Variables related to the treatment and results are summarized in Table 2. The associated visceral resections 
Table 2. Treatment and Results - (Continued)

\begin{tabular}{|c|c|}
\hline & $\mathrm{n}=\mathbf{3 4}$ \\
\hline \multicolumn{2}{|l|}{ Cytoreduction } \\
\hline $\mathrm{CCO}$ & $27(79 \%)$ \\
\hline $\mathrm{CC} 1$ & $4(12 \%)$ \\
\hline $\mathrm{CC} 2$ & $2(6 \%)$ \\
\hline $\mathrm{CC} 3$ & $1(3 \%)$ \\
\hline \multicolumn{2}{|l|}{ Cytoreducion $\mathrm{CCO}$} \\
\hline Primary & $19(70 \%)$ \\
\hline Relapse & $8(30 \%)$ \\
\hline \multicolumn{2}{|l|}{ Cytoreduction CC1 } \\
\hline Primary & $2(50 \%)$ \\
\hline Relapse & $2(50 \%)$ \\
\hline \multicolumn{2}{|l|}{ Cytoreduction CC2 } \\
\hline Primary & $2(100 \%)$ \\
\hline Relapse & 0 \\
\hline \multicolumn{2}{|l|}{ Cytoreduction CC3 } \\
\hline Primary & 0 \\
\hline Relapse & $1(100 \%)$ \\
\hline Complications & $19(56 \%)$ \\
\hline Digestive fistulas & $3(9 \%)$ \\
\hline Reoperation & $6(18 \%)$ \\
\hline Death 90 days & $2(6 \%)$ \\
\hline Stay in ICU & $6(2-36)$ \\
\hline Postoperative stay & $22(8-63)$ \\
\hline Stay $>20$ days & $20(59 \%)$ \\
\hline Primary & $27(10-56)$ \\
\hline Relapse & $14(8-63)$ \\
\hline
\end{tabular}

Quantitative variables: median (range). Nominal variables: count (\%).

are shown in Table 3.

All the digestive fistulas corresponded to the leakage of colorectal anastomosis. The re-operations correspond to the three fistulas and to three eviscerations. Deaths correspond to liver failure and to secondary sepsis to necrotizing fasciitis of the abdominal wall.

Those who suffer postoperative complications of grade II/ III are of Clavien-Dindo 19 (56\%) cases. The most frequent were of a septic type: a pleural and pulmonary type, abdominal collections, and those related to the incision. Complications are associated with major or minor extension of the surgery; nonetheless, they are not associated with the presence of comorbidity $(\mathrm{P}=0.886)$ nor with age $(\mathrm{P}=0.846)$.

In this way, the average IPC in complicated cases is from $16 \pm 8$ against $10 \pm 8$ in those without complications $(\mathrm{P}=$ 0.026). A major number of complications per patient also are associated with the IPC $(\mathrm{P}=0.018)$. Moreover, in complicated cases, there is an average of removed viscera of $5 \pm 3$, while in non-complicated cases, there is an average of $2 \pm 2$. A large number of complications per patient are also associated with
Table 3. Visceral Resections in Order of Frequency

\begin{tabular}{lll}
\hline Organ & $\mathbf{n}$ & $\mathbf{\%}$ \\
\hline Sigmoid colon & 18 & 53 \\
Small intestine & 14 & 41 \\
Cecum and appendiz & 13 & 38 \\
Gallbladder & 12 & 35 \\
Rectum & 11 & 32 \\
Spleen & 11 & 32 \\
Transverse colon & 7 & 21 \\
Ascending colon & 5 & 15 \\
Pancreas body-tail & 5 & 15 \\
Hepatic peritoneum & 5 & 15 \\
Partial bladder & 4 & 12 \\
Descending colon & 4 & 12 \\
Liver & 4 & 12 \\
Diaphragm & 4 & 12 \\
Total bladder & 3 & 9 \\
Ureter & 3 & 9 \\
Major vessels & 3 & 9 \\
Stomach & 2 & 6 \\
Kidney & 2 & 6 \\
Adrenal & 2 & 6 \\
Total & 30 cases & \\
\hline
\end{tabular}

the number of removed viscera $(\mathrm{P}=0.001)$.

Medium follow-up is 7 months (interquartile range: 3.7 - 10.2). Medium general survival is 16 months, while the medium survival rate free of cancer is 15 months. As can be observed (Fig. 2), relapses begin to appear after 6 months. Even though no significant statistical differences (log-rank: $\mathrm{P}=$ 0.663 ) are found in the event of relapses, it is useful to compare cases with $\mathrm{CCO}$ and non-CCO; possibly due to the short follow-up time period, the medium survival rate free of illness was almost double when $\mathrm{CCO}$ is compared to non-CCO (15 months versus 8 months). No patient received HIPEC. In $22(65 \%)$ cases postoperative chemotherapy was administered with a medium delay of 2 months $(0.4-8)$.

\section{Discussion}

The Multidisciplinary Unit of Abdominal Pelvic Oncology Surgery (MUAPOS) was created at our institution in January 2013, dedicating itself entirely to the treatment of advanced ovarian cancer by incorporating extensive surgical techniques for cytoreduction of advanced ovarian and peritoneal cancers. This multidisciplinary focus of the technique used has reached an improvement in the percentage of optimal achieved cytoreduction. Thus, in our retrospective series of 34 patients for whom a CS for advanced ovarian cancer was performed, we statistically analyzed the results obtained in terms of effective- 


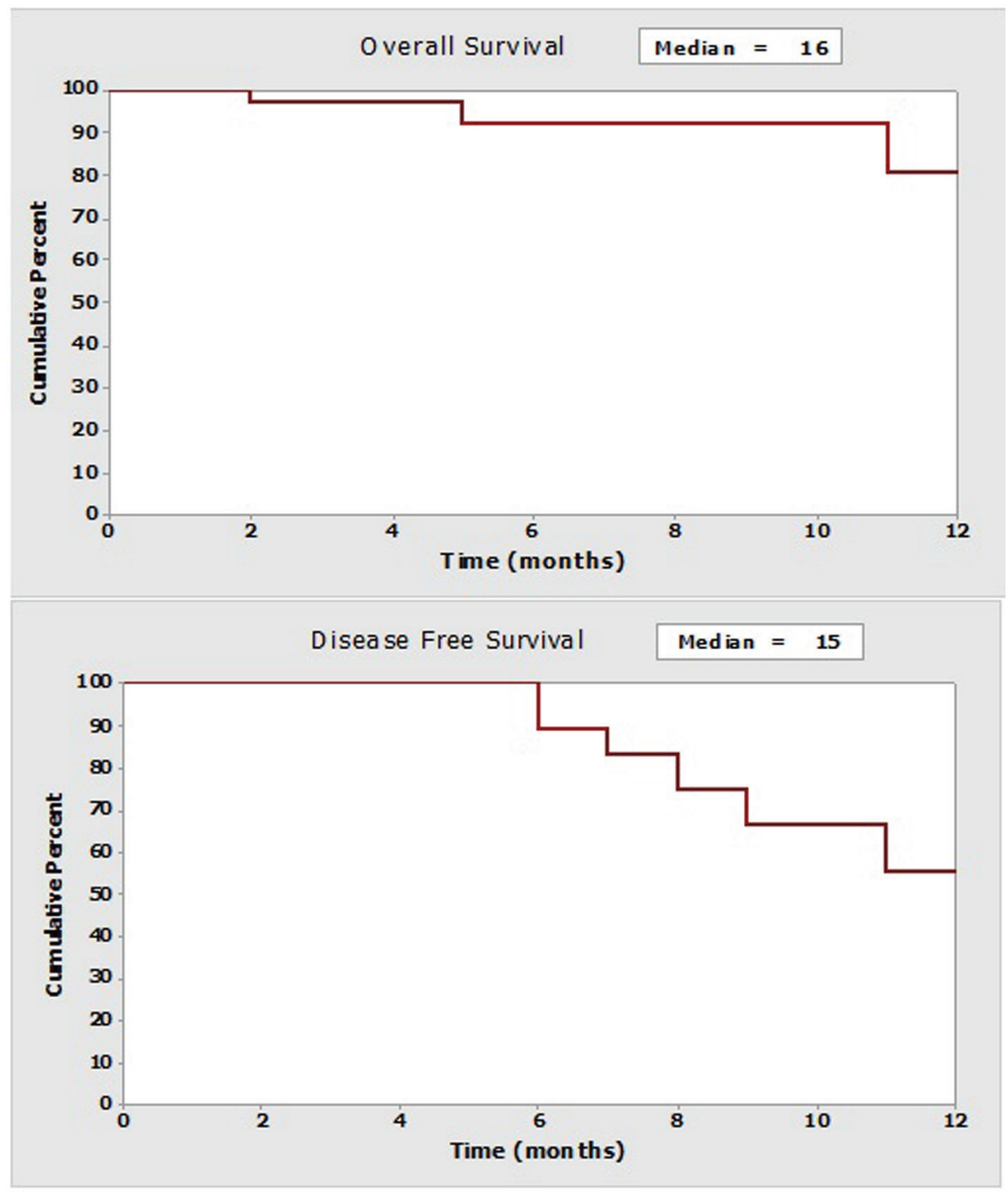

Figure 2. Curves of survival at the follow-up year (Kaplan-Meier).

ness as well as complications due to the surgery.

Optimal cytoreduction at the time of the first surgery has shown to be the most important prognostic factor related to the increase in survival of patients with epithelial cancer of the ovary $[10,11]$. In our retrospective series of patients with elevated tumor amounts and stages III and IV of the disease, a complete cytoreduction (CC0) was achieved in 79\% of the cases and an optimal cytoreduction (CC0 or $\mathrm{CC} 1$ ) in $91 \%$ of the cases. The mean operating IPC was 12 (2 - 33), being in $62 \%$ of the cases above 10 . These numbers demonstrate, just as it happens in other works, that optimal cytoreduction can achieve a high percentage rate in cases even though the tumor amount may be high and even though they may be found at stage IV of the disease $[10,12]$. Some studies have shown that centers with an optimal cytoreduction rate $>75 \%$ provide a $50 \%$ mean survival rate compared to centers that only offer a $25 \%$ optimal cytoreduction rate. Nevertheless, it is also known that an optimal cytoreduction above $50 \%$ requires the incor- poration of extensive surgical techniques in the superior abdomen and can require numerous visceral resections $[10,13]$. In our series visceral resections were carried out in 30 patients $(88 \%)$, the sigmoid colon forming part of the pelvic peritonectomy being the most frequent.

The percentage of patients with neoadjuvant in primary tumors was $26.4 \%$. We agree with some other investigators that the effect of the fibrosis on the tumor after having administered chemotherapy can make interval surgery more difficult and even could hide areas affected by the tumor [14]. Moreover, some authors have demonstrated that the best survival and the best interval free of the disease is obtained when an optimal primary CS is carried out and that each chemotherapy cycle added before surgery reduces the percentage of survival [15].

Regulated pelvic and aortic lymphadenectomy in cases of advanced ovarian cancer reveal a high affectation of the lymph nodes, approximately $40-78 \%$, and this affectation can be of a microscopic type in $35 \%$ of cases $[16,17]$. In our series a 
regulated lymphadenectomy was carried out in 27 cases $(80 \%)$ finding a mean of affected lymph nodes of $(0-36)$. Only in cases without lymph node affectation, neither deaths nor relapses occurred amongst our patients, and even though there was no statistical significance due to the scarce number of cases and follow-up time, the tendency in the series seems evident to us. Nevertheless, one will have to await the conclusion of the German study LION in order to know the real role of lymphadenectomy in advanced ovarian cancer.

Our results reflect $56 \%$ of moderate/severe complications according to the classification of Clavien-Dindo and a $6 \%$ mortality rate, similar to other published series [18]. The introduction of extensive surgery within our center required the creation of a multidisciplinary team composed by surgeon specialists from different medical fields (general surgery, vascular, gynecologic oncology and urology), who have abundant experience in their respective areas, but lack knowledge in terms of cooperative approach to advanced ovarian cancer. This lack of experience could be the reason for the high complication rate that the group exposes; however, it should improve over time.

Nonetheless, in our results, contrary to what happens in other series [19], associated comorbidity and age are not associated with a large number of complications. On the other hand, as would seem logical, high morbidity is associated with high surgical aggressiveness. This is demonstrated by the larger number of complications associated with a higher measure of the tumor (ICP > 10). Such cases require more surgical effort in order to achieve a $\mathrm{CCO}$ and, for the same reason, more than two viscera dried up in the same patient.

Kuhn et al [13] analyze the influence of extensive surgery in the superior abdominal region as part of the $\mathrm{CC}$ of advanced ovarian cancer and they reach the conclusion that mortality and morbidity increased in patients on whom extensive superior abdominal surgery had been performed, including procedures such as cholecystectomy, splenectomy, distal pancreatectomy, diaphragmatic surgery, hepatectomies, etc. As a result, the authors question the risk/benefit of this type of surgery. Nevertheless they also observe that patients who have had superior abdominal surgery in which a microscopic residual tumor was removed lived a mean of 71 months compared to a 15-month mean for those who lived with a residual macroscopic tumor.

Eisenkop [20] demonstrates that there does not exist a concrete location of the tumor or a specific surgical maneuver that correlates to the biological aggressiveness of the tumor, and that, therefore, a cytoreduction procedure should not be modified nor finalized simply because it affects a specific organ or area. Furthermore, nowadays the fact is no longer questioned that in terms of survival a surgery of suboptimal cytoreduction will not benefit affected patients with advanced ovarian cancer $[15,21]$. The extent of surgery in patients with advanced ovarian cancer should thus be determined by the performance status and the capacity of the patient to tolerate this very aggressive surgery, as well as by the capacity of a competent surgical team to carry it out with diligence, more than by reason of ages, location, or extent of the illness present at the time of surgery. In spite of the short follow-up time period in these patients, the results obtained are in line with other published studies, and although some results may not be statistically significant, upon comparing complete cytoreduction surgeries with those that were not, a tendency is found towards a gained benefit in terms of intervals free of disease, as has been the case in studies previously cited [22].

In conclusion, this study has shown how CS from advanced ovarian cancer results to be aggressive and complex if we want to obtain a CCO; for this reason, specialist teams should be prepared to cope with a high rate of complications, at least during their initial stages. Through the adequate process of patient selection and the acquisition of a greater experience in the cooperative treatment of advanced ovarian cancer, overall results for this type of surgery should experience a rapid increase, in terms of efficiency and safety.

\section{Acknowledgement}

The authors would like to thank Michelle Fuerch and Marta Llueca for their editorial assistance in the preparation of the manuscript.

\section{Author Note}

MUAPOS Working Group (Multidisciplinary Unit of Abdominal Pelvic Oncology Surgery): Salvador-Sanchis JL, CalpeGomez E, Jativa-Porcar R, Moreno-Clari E, Montanes-Pauls B, Queralt-Martin R, Villarin-Rodriguez A, Granel-Villach L, Lluch-Martin B, Villegas-Canovas C, Angel-Yepes V, MoyaSanz A, Medina-Medina C, Delgado-Barriga K, RodrigoAliaga M, Ruiz N, Lopez A, Oliva C, Colecha M, Vizcaino J, Guijarro M, Canete A, Estelles D, Maazouzi Y, Piquer L.

\section{References}

1. Pecorelli S, Odicino F, Maisonneuve P, Creasman W, Sheperd J, Sideri M, Benedet J. Carcinoma of the ovary. J Epidemiol Biostat. 1988;3:75-102.

2. Saitou M, Iida Y, Komazaki H, Narui C, Matsuno K, Kawabata A, Ueda K, et al. Success rate and safety of tumor debulking with diaphragmatic surgery for advanced epithelial ovarian cancer and peritoneal cancer. Arch Gynecol Obstet. 2015;291(3):641-646.

3. Llueca JA, Martinez-Ramos D, Escrig-Sos J, Torrella-Ramos A, HerraizJL,et al. Current status of ovarian cancer in the Spanish Province of Castellon. Prognostic factors in observed and relative survival. A population cancerregistry-based study between 2004 and 2008.Prog Obstet Ginecol. 2014;57:405-412.

4. Serov SF, Scully RE, Sorbin LH. Histological typing of ovarian tumors. In: International histological classification of tumors. No. 9. Geneva: World Health Organization; 1973. p.17.

5. Day TG, Jr., Gallager HS, Rutledge FN. Epithelial carcinoma of the ovary:prognostic importance of histologic grade. Natl Cancer Inst Monogr. 1975;42:15-21.

6. Changes in definitions of clinical staging for carcinoma of the cervix and ovary: International Federation 
of Gynecology and Obstetrics. Am J Obstet Gynecol. 1987;156(1):263-264.

7. Esquivel J, Farinetti A, Sugarbaker PH. Elective surgery in recurrent colon cancer with peritoneal seeding: when not to proceed. Geka Chiryo. 1999;20:81.

8. Morrow CP, Curtin JP. Surgery for ovarian neoplasia. In: Morrow CP, Curtin JP, editors. Gynecologic cancers surgery. California; 2013. p. 857-892.

9. Dindo D, Demartines N, Clavien PA. Classification of surgical complications: a new proposal with evaluation in a cohort of 6336 patients and results of a survey. Ann Surg. 2004;240(2):205-213.

10. Eisenkop SM, Friedman RL, Wang HJ. Complete cytoreductive surgery is feasible and maximizes survival in patients with advanced epithelial ovarian cancer: a prospective study. Gynecol Oncol. 1998;69(2):103-108.

11. Hoskins WJ, McGuire WP, Brady MF, Homesley HD, Creasman WT, Berman M, Ball H, et al. The effect of diameter of largest residual disease on survival after primary cytoreductive surgery in patients with suboptimal residual epithelial ovarian carcinoma. Am J Obstet Gynecol. 1994;170(4):974-979; discussion 979-980.

12. Piver MS, Baker T. The potential for optimal (less than or equal to $2 \mathrm{~cm}$ ) cytoreductive surgery in advanced ovarian carcinoma at a tertiary medical center: a prospective study. Gynecol Oncol. 1986;24(1):1-8.

13. Kuhn W, Florack G, Roder J. The influence of upper abdominal surgery on perioperative morbidity and mortality in patients with advanced ovarian cancer FIGO III and IV. Int J Gynecol Cancer. 1998;8:56-63.

14. Vergote I, Trope CG, Amant F, Kristensen GB, Ehlen T, Johnson N, Verheijen RH, et al. Neoadjuvant chemotherapy or primary surgery in stage IIIC or IV ovarian cancer. N Engl J Med. 2010;363(10):943-953.

15. Bristow RE, Tomacruz RS, Armstrong DK, Trimble EL,
Montz FJ. Survival effect of maximal cytoreductive surgery for advanced ovarian carcinoma during the platinum era: a meta-analysis. J Clin Oncol. 2002;20(5):1248-1259.

16. Morice P, Joulie F, Camatte S, Atallah D, Rouzier R, Pautier P, Pomel C, et al. Lymph node involvement in epithelial ovarian cancer: analysis of 276 pelvic and paraaortic lymphadenectomies and surgical implications. J Am Coll Surg. 2003;197(2):198-205.

17. Di Re F, Baiocchi G., Fontanelli R. Systematic pelvic and paraaorticlymphadenctomy for advanced ovarian cancer: prognostic significance of node metastasis. GynecolOncol. 1996;62:330-335.

18. Chi DS, Franklin CC, Levine DA, Akselrod F, Sabbatini $\mathrm{P}$, Jarnagin WR, DeMatteo R, et al. Improved optimal cytoreduction rates for stages IIIC and IV epithelial ovarian, fallopian tube, and primary peritoneal cancer: a change in surgical approach. Gynecol Oncol. 2004;94(3):650-654.

19. Corman ML. Carcinoma of the rectum. In: Corman ML, editor. Colon and rectal surgery. 4th ed. Philadelphia: Lippincott-Raven; 1998. p. 733-862.

20. Eisenkop SM, Spirtos NM, Friedman RL, Lin WC, Pisani AL, Perticucci S. Relative influences of tumor volume before surgery and the cytoreductive outcome on survival for patients with advanced ovarian cancer: a prospective study. Gynecol Oncol. 2003;90(2):390-396.

21. Chi DS, Liao JB, Leon LF, Venkatraman ES, Hensley ML, Bhaskaran D, Hoskins WJ. Identification of prognostic factors in advanced epithelial ovarian carcinoma. Gynecol Oncol. 2001;82(3):532-537.

22. Luyckx M, Leblanc E, Filleron T, Morice P, Darai E, Classe JM, Ferron G, et al. Maximal cytoreduction in patients with FIGO stage IIIC to stage IV ovarian, fallopian, and peritoneal cancer in day-to-day practice: a Retrospective French Multicentric Study. Int J Gynecol Cancer. 2012;22(8):1337-1343. 